\title{
COMPARISON OF LIGHT QUARK AND CHARM QUARK FRAGMENTATION
}

\author{
P. KESTEN, C. AKERLOF, G. BONVICINI, J. CHAPMAN, D. ERREDE, N. HARNEW ' \\ D.I. MEYER, D. NITZ, A.A. SEIDL ${ }^{2}$, R. THUN, T. TRINKO ${ }^{2}$, M. WILLUTZKY \\ University of Michigan, Ann Arbor, MI 48109, USA
}

M. DERRICK, P. KOOIJMAN, J.S. LOOS, B. MUSGRAVE, L.E. PRICE, J. SCHLERETH, K. SUGANO, J.M. WEISS ${ }^{3}$, D.E. WOOD ${ }^{2}$

Argonne National Laboratory, Argonne, IL 60439, USA

P. BARINGER, D. BLOCKUS, B. BRABSON, S.W. GRAY ${ }^{4}$, C. JUNG, H. NEAL, H. OGREN, D.R. RUST, M. VALDATA-NAPPI ${ }^{5}$

Indiana University, Bloomington, IN 47405, USA

S. ABACHI, I. BELTRAMI, B.G. BYLSMA, R. DeBONTE, K.K. GAN, F.J. LOEFFLER, E.H. LOW, R.L. McILWAIN, D.H. MILLER, C.R. NG, L.K. RANGAN, E.I. SHIBATA

Purdue University, West Lafayette, IN 47907, USA

and

B. CORK

Lawrence Berkeley Laboratory, Berkeley, CA 94720, USA

Received 23 July 1985

\begin{abstract}
Separate samples of charm quark and light quark $(u, d, s)$ jets have been isolated in an experiment studying $\mathrm{e}^{+} \mathrm{e}^{-}$ annihilations at $\sqrt{s}=29 \mathrm{GeV}$. The results come from data corresponding to an integrated luminosity of $111 \mathrm{pb}^{-1} \mathrm{collected} \mathrm{by}$ the High Resolution Spectrometer. Differences were observed in charged multiplicities, momentum distributions, and rapidity of the size expected from the different fragmentation functions and leading particle decay properties of the two samples.
\end{abstract}

In this paper we report the first comparison of the fragmentation properties of the charmed quark (c) with light quarks $(\mathrm{u}, \mathrm{d}, \mathrm{s})$. The data are selected from a sample of $\sim 40 \mathrm{~K}$ hadronic events collected by the

1 Present address: CERN, CH-1211 Geneva 23, Switzerland.

2 Present address: Lockheed Missiles \& Space Co., Sunnyvale, CA 94086, USA.

${ }^{3}$ Present address: SRI International, Menlo Park, CA 94025, USA.

${ }^{4}$ Present address: Laboratory for Nuclear Studies, Cornell University, Ithaca, NY 14853, USA.

5 Permanent address: INFN, I-56010 Pisa, Italy. high resolution spectrometer (HRS) at the PEP $\mathrm{e}^{+} \mathrm{e}^{-}$ colliding beam facility, corresponding to an integrated luminosity of $111 \mathrm{pb}^{-1}$. The storage ring was operated at a center-of-mass energy of $\sqrt{s}=29 \mathrm{GeV}$.

The HRS is a general-purpose detector with outstanding momentum resolution for charged tracks [1]. Photons and electrons are also measured in a set of calorimeters that surround the tracking system and cover $90 \%$ of the full șolid angle. A set of threshold Cerenkov counters with $\pi$ and $K$ thresholds of $1.5 \mathrm{GeV} / c$ and $6 \mathrm{GeV} / c$, respectively, were in operation for much of the data-taking. 
The charm quark data sample is specified by the observation of a $\mathrm{D}^{*+} \rightarrow \mathrm{D}^{0} \pi^{+}$decay (plus charge conjugate) followed by one of the decays: $\mathrm{D}^{0} \rightarrow \mathrm{K}^{-} \pi^{+}$, or $\mathrm{D}^{0} \rightarrow \mathrm{K}^{-} \pi^{+} \pi^{+} \pi^{-}$. The energy fraction $Z_{\mathrm{D}^{*}}$ $=2 E_{\mathrm{D}^{*}} / \sqrt{s}$ was required to be $>0.4$ for the former decay mode and $>0.5$ for the latter. To be called a kaon a particle had to go undetected in the Cerenkov counter system. The $D^{*}-D^{0}$ mass difference $(\Delta M)$ shows a narrow peak containing $150 \mathrm{D}$ *'s [2], which we use to tag c $\overline{\mathrm{c}}$ events. We estimate a $(15 \pm 3) \%$ light quark contamination in the charm quark data sample after applying the mass difference selection of 144 $\mathrm{MeV}<\Delta M<147 \mathrm{MeV}$. The high $Z_{\mathrm{D}^{*}}$ requirement also effectively excludes those events originating from $b \bar{b}$ production. The momentum spectrum of the $D^{*}$ trigger particle is shown in fig. $1 \mathrm{a}$.
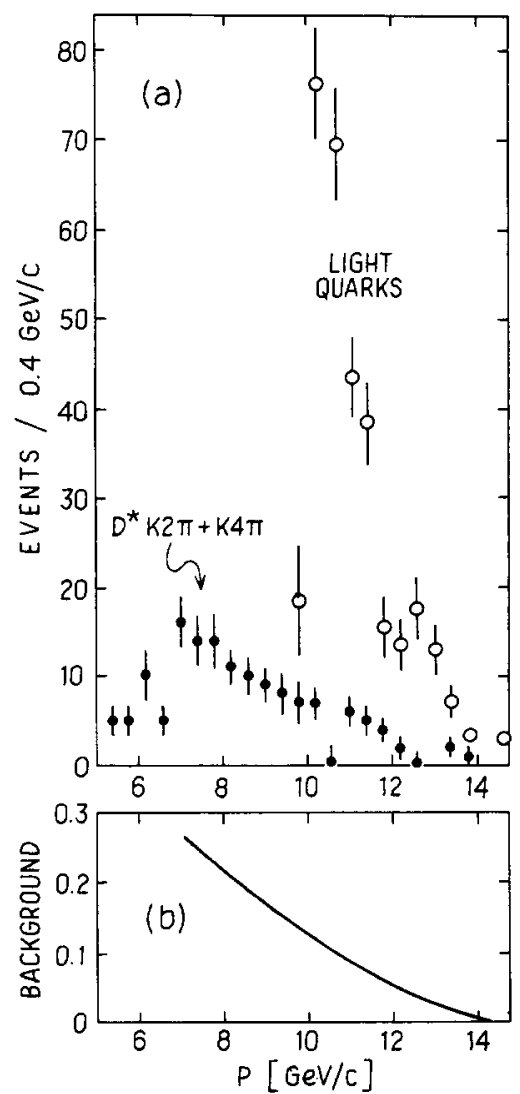

Fig. 1. (a) Momentum spectrum of trigger $D^{*}$ meson and trigger particle for light quark jet selection. (b) Monte Carlo prediction of charmed quark jet contamination in light quark sample.
The light quark sample was obtained by selecting all events containing one charged trigger particle with momentum fraction $Z=2 p / \sqrt{s}$ greater than 0.7 $(p>10.15 \mathrm{GeV} / c)$. Even though the $\mathrm{c}$ and $\mathrm{b}$ quark fragmentation is hard, the events have few high-momentum $\pi$ and $\mathrm{K}$ mesons in the final state because of the large decay multiplicity of $\mathrm{D}$ and $\mathrm{B}$ mesons. The momentum spectrum of the trigger particle is shown in fig. 1a. There are 314 events above the $10.15 \mathrm{GeV} /$ $c$ momentum cut. The excellent momentum resolution of the HRS is important in allowing a clean selection of such high momentum charged particles. As shown by the line, which was calculated from a Monte Carlo (MC) simulation of the experiment, the data include an estimated background from $c \bar{c}$ and $\mathrm{b} \overline{\mathrm{b}}$ events of $(11 \pm 2) \%$. (In the MC simulation, the primary partons are generated according to the Lund model and the fragmentation of the partons into hadrons uses the Feynman and Field prescription [3]. The charm fragmentation function is adjusted to agree with our measured $D^{*}$ data [2]. We also use this simulation to correct the measured quantities for the detector acceptance and resolution.)

For both data sets the thrust axis of the event was required to be inclined at greater than $50^{\circ}$ with respect to the $\mathrm{e}^{+} \mathrm{e}^{-}$beam direction, and the charged multiplicity of the event $\left(N_{\mathrm{ch}}\right)$ to be greater than five. For the charm quark events, we count the decay products of the observed $D^{*}$ as a single particle.

The characteristics of the jets opposite to the trig. ger jet are compared. For two-jet events long range jet -jet correlations are weak so that the fragmentation of the opposite jet should proceed independently of the trigger jet [4]. A plane perpendicular to the thrust axis was used to divide the events into two jets. For three-jet events, however, the requirement of a high-momentum trigger particle tends to suppress light quark events with hard gluons compared to charm quark events. While the hard gluon events account for less than $10 \%$ of both samples, they can have a strong effect on averages of jet variables. To remove those three-jet events which would be suppressed in different amounts in the two samples, we required that $M^{2} / s<0.04$ for the jet opposite to the trigger, where $M$ is the jet mass calculated from the charged particles, assuming the pion mass [5]. As seen in the MC results of fig. 2 , this selection has little effect on the two-jet events but cuts a significant frac- 


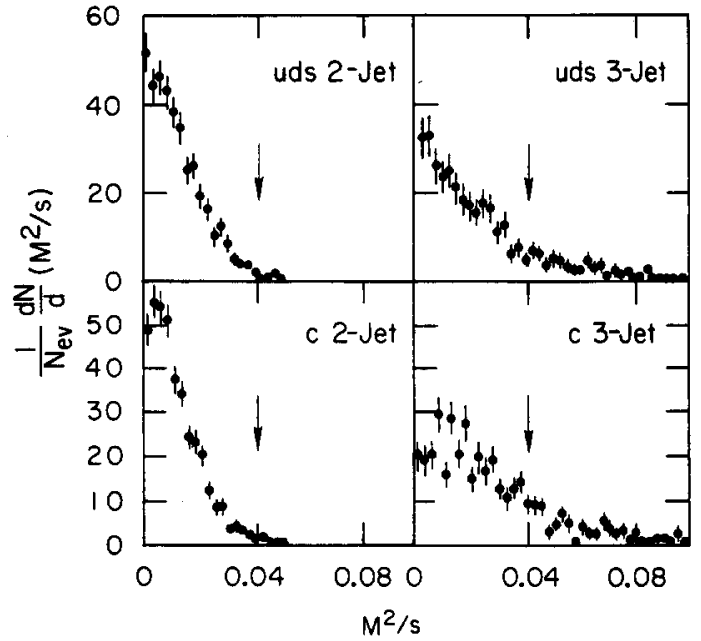

Fig. 2. $\mathrm{M}^{2} / \mathrm{s}$ distributions calculated from the MC simulation for charm quark and light quark fragmentation for two-jet $(q \bar{q})$ and three-jet ( $q \bar{q} g$ ) events for the jet opposite the trigger jet.

tion of the three-jet events. The final data set contains $111 \mathrm{cc}$ events and 287 light quark events.

The mean values of the kinematic variables measured in the jet opposite the trigger jet, corrected for detector acceptance, are given in table 1 . There are statistically significant differences in some variables for the two samples which disappear when account is taken of the different kinematic behavior of the leading quarks.

The mean multiplicity of the charm quark jets is higher than that of the light quark jets. Although the higher momentum of the leading particle in the $c$ quark events leads to a lower multiplicity for the sea fragmentation, this effect is more than compensated by the decay multiplicity of the $\mathrm{c}$ quark mesons. A detailed calculation [5] uses the branching ratios to obtain the mean number of charged decay products of charmed and light quark leading particles. The fragmentation functions are used to obtain the resi- dual energy ( $\left.E_{\text {Beam }}-E_{\text {leading quark }}\right)$. The measured charged particle multiplicity in $\mathrm{e}^{+} \mathrm{e}^{-}$collisions at this residual energy (corrected because no charm particles are produced in the hadronization of the residual energy) and the mean number of charged decay products gives $\left\langle N_{\mathrm{ch}}\right\rangle_{\mathrm{calc}}=6.4$ for chark quarks and 5.7 for light quarks which is in good agreement with the observed values in table 1 . Suitably weighted, the $\left\langle N_{\mathrm{ch}}\right\rangle$ measurements are consistent with our inclusive jet sample ${ }^{\ddagger 1}$. The multiplicity distributions, normalized to the mean value $N_{\mathrm{ch}} /\left\langle N_{\mathrm{ch}}\right\rangle$, are quite similar as seen in fig. $3 \mathrm{a}$.

The values of $\left\langle p_{\mathrm{T}}\right\rangle$ for charged particles in the two samples are the same, within errors, as are the $p_{\mathrm{T}}^{2}$ distributions shown in fig. $3 \mathrm{~b}$. They are also in agreement with $\left(p_{\mathrm{T}}\right)$ for charged particles measured for the inclusive sample [6].

The source of the difference in $\langle p\rangle$ for charged particles from light and charm quark jets as seen in table 1 is the same as that for $\left\langle N_{\mathrm{ch}}\right\rangle$. The $p$ distributions for the two samples are shown in fig. 3c.

The rapidity distributions of fig. 4 show a $10 \%$ higher plateau value for the charm quark jets, consistent with the higher multiplicity of that sample. In calculating rapidity, we assign the pion mass to all particles. The rapidity plateau is somewhat wider for the light quark events (fig. 4a); the distribution falls to $\left(1 / N_{\mathrm{ev}}\right) \mathrm{d} N / \mathrm{d} Y=1$ at $Y=2.8 \pm 0.1$ for the charm jets and $Y=3.0 \pm 0.1$ for the light quark jets.

The MC curves on fig. 4 show the predicted distributions in rapidity of the two components: the dash-dotted curves correspond to the decay particles from the hadron that contains the leading quark, whereas the dashed curve shows the rapidity distribution of the remaining particles in the jet. The sum of

\footnotetext{
\$1 We have previously reported values for the inclusive jet sample of $\left\langle N_{\mathrm{ch}}\right\rangle=6.55 \pm 0.30,\langle p\rangle=1.31 \pm 0.02 \mathrm{GeV} / c$, $\left\langle p_{\mathrm{T}}\right\rangle=0.380 \pm 0.005 \mathrm{GeV} / c$ and $\langle S\rangle=0.130 \pm 0.003$ \pm 0.010 [6].
}

Table 1

Mean values of jet variables

\begin{tabular}{llllll}
\hline & $\left\langle N_{\mathrm{ch}}\right\rangle$ & $\langle p\rangle \mathrm{GeV} / c$ & $\left\langle p_{\mathrm{T}}\right\rangle \mathrm{GeV} / c$ & $\langle S\rangle$ & $\langle 1-T\rangle$ \\
\hline charm quark jets & $6.6 \pm 0.2$ & $1.38 \pm 0.06$ & $0.39 \pm 0.01$ & $0.094 \pm 0.010$ & $0.082 \pm 0.005$ \\
light quark jets & $5.8 \pm 01$ & $1.52 \pm 0.04$ & $0.40 \pm 0.01$ & $0.087 \pm 0.007$ & $0.092 \pm 0.004$ \\
\hline
\end{tabular}



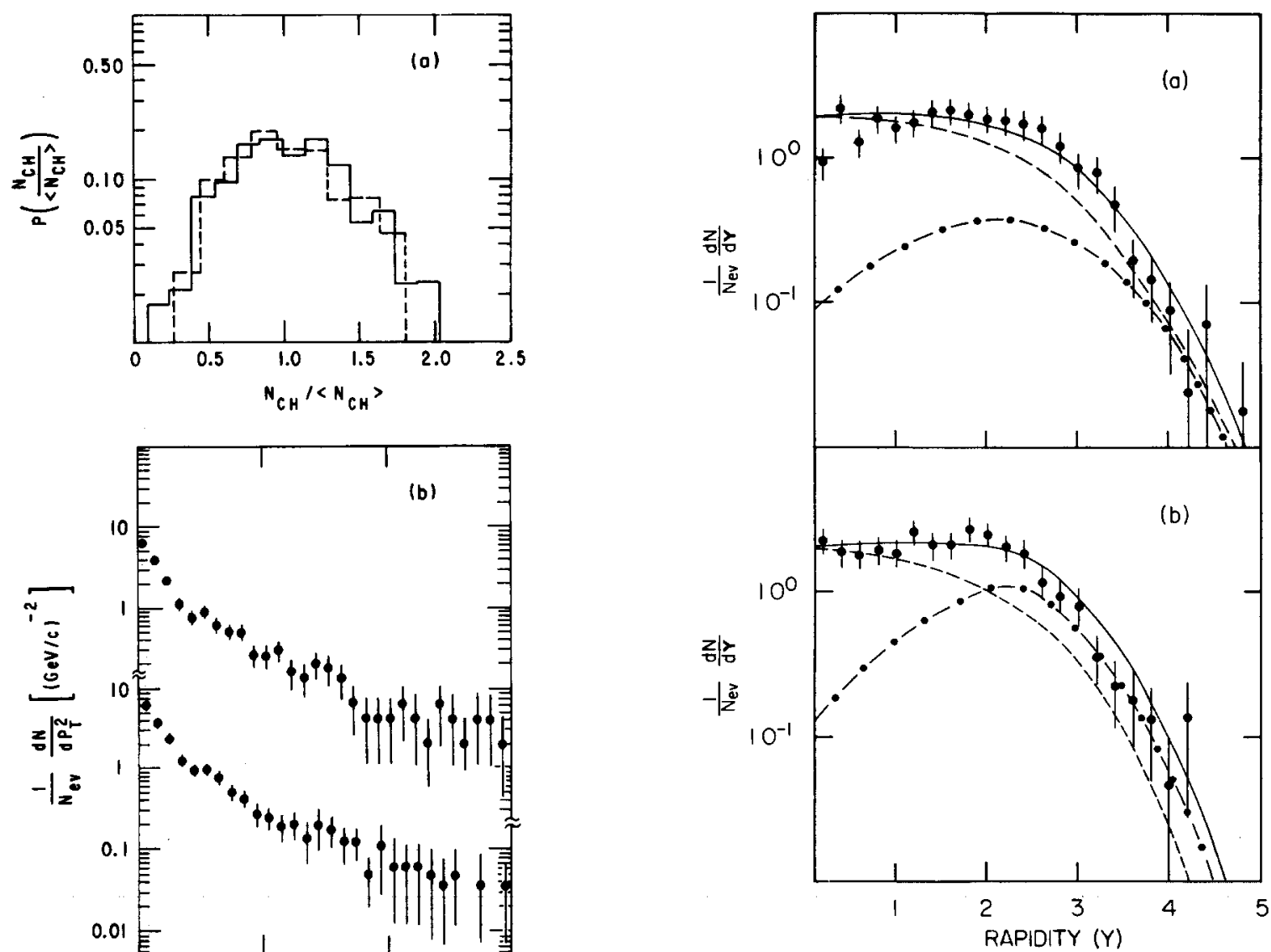

Fig. 4. Rapidity distributions for (a) u, d, s quark events, (b) c quark events. The dash-dotted line shows the distribution of the decay products of the primary meson, and the dashed line the mesons from the sea quarks according to the MC simulation of the experiment.

these two components gives the full curve(s). No attempt has been made to adjust the parameters of the MC simulation to fit the data beyond the overall normalization. The peaking near $Y=2$ results from the decay products of the leading particle which is strong. er for the charm quark than for the light quark because of the higher D meson decay multiplicity and

Fig. 3. Comparison of (a) charged multiplicity distributions of the jets opposite the trigger jet for c quarks (full histogram) and $u, d, s$ quarks (dashed histogram), (b) $p_{\mathrm{T}}^{2}$, and (c) single particle momentum distributions. In (b) and (c) the upper data is for $c$ quarks and the lower data for the $u, d, s$ quark sample. 
the harder charm quark fragmentation. The effect is even more pronounced for the $b$ quark as has been reported by the DELCO collaboration [7]. Such differences might be useful to select top quark jets in future studies of very high energy $\mathrm{e}^{+} \mathrm{e}^{-}$or hadronic interactions [8].

The TASSO group [9] has compared properties of charm quark jets (selected with a $D^{*}$ tag) with a global data sample and observed no differences. With a larger data sample and a selected light quark sample the present experiment detects some differences between light and charm quark events, but these are attributable to effects associated with the difference in mass of the leading quarks. No other differences between hadronization of quarks of different flavors is observed.

This work was supported by the US Department of Energy. We thank the technical staff of PEP and all the collaborating institutions for making the experiment possible.

\section{References}

[1] D. Bender et al., Phys. Rev. D30 (1984) 515.

[2] M. Derrick et al., Phys. Lett. 146B (1984) 261.

[3] B. Andersson, G. Gustafson, G. Ingelman and T. Sjöstrand, Phys. Rep. 97 (1983) 31;

R.D. Field and R.P. Feynman, Nucl. Phys. B136 (1978) 1.

[4] TASSO Collab., R. Brandelik et al., Phys, Lett. $100 \mathrm{~B}$ (1981) 357;

C. Berger et al., Nucl. Phys. B214 (1983);

M. Nappi, XIV Intern. Symp. on Multiparticle dynamics, eds. P. Yager and J. Gunion (Lake Tahoe, CA, 1983) p. 75.

[5] P. Kesten, Ph.D. Thesis, University of Michigan (1985).

[6] D. Bender et al., Phys. Rev. D31 (1985) 1.

[7] DELCO Collab., M. Sakuda et al., Phys. Lett 152B (1985) 399.

[8] T.D. Gottschalk and T. Sjöstrand, Rapidity signals for top, Proc. 1984 Summer Study on the Design and utilization of the superconducting super collider (Snowmass, $\mathrm{CO}$, 1984) p. 81.

[9] TASSO Collab., M. Althoff et al, Phys. Lett. 135B (1984) 243. 